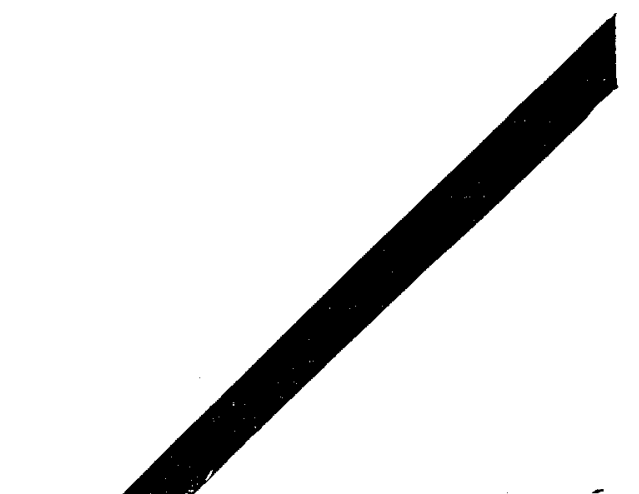

\title{
RÉGIME DE TARISSEMENT DE LA FOUX-DE-LA-VIS (GARD)
}

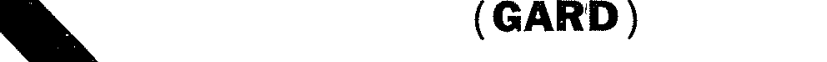

ervos entumamanase.

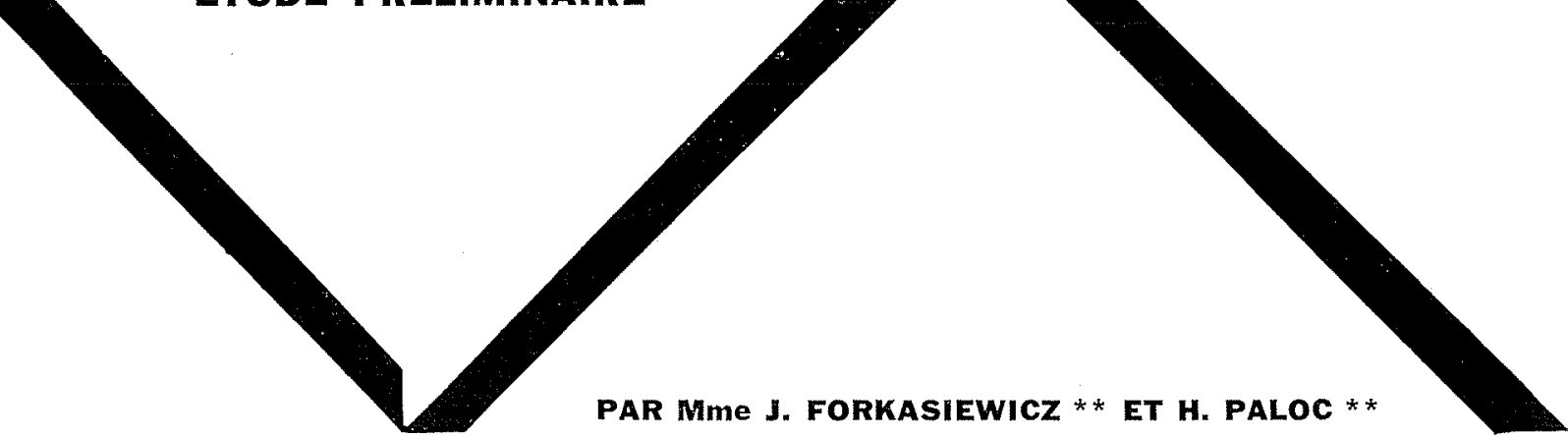

\section{Avant-propos}

Le présent travail est un résumé de la communication présentée au Colloque sur l'hydrogéologie des roches fissurées organisé à Dubrovnik, du 7 au 15 octobre 1965 et à la séance du 17 novembre 1966 de la Société Hydrotechnique de France consacrée à l'hydraulique des terrains fissurés.

\section{Introduction}

La Foux-de-la-Vis est la plus importante résurgence de la région constituant les Grands Causses du Massif Central français, et l'une des plus grosses sources de France. Son bassin se développe dans les calcaires et dolomies jurassiques des causses méridionaux. C'est une source pérenne, bien que cette pérennité ait été interrompue quatre fois depuis 1779 .

Son débit d'étiage est élevé, rarement inférieur à $1 \mathrm{~m}^{3} / \mathrm{s}$ et son débit de crue parait dépasser $10 \mathrm{~m}^{3} / \mathrm{s}$. La station de jaugeage se trouve à $4 \mathrm{~km}$ en aval de la source, mais aucun apport ni aucune perte ne se

Le travail dont le résumé est publié ici a été présenté comme communication au collogue sur l'hydrologie des roches fissurées organisé à Dubrovnili (Yougoslavie), en octobre 1965, par l'U.N.E.S.C.O., avee le concours de l'A.I.H.S. et de I'A.I.H.

Il a été publié in extenso:

- dans le rapport B.R.G.M., DS 65 A 84, octobre 1965, Paris;

- dans le compte rendu du Colloque sur l'hydrogeologie des

loches fissurées a Dubrovnil, octobre 1965, A.I.H.S.

* B.R.G.M., Service Hydrosélogie. produisant entre ces deux points, les débits mesurés peuvent ètre confondus avec ceux de la source. Ayant à notre disposition les relevés des débits moyens journaliers pour la période du 1 er janvier 1950 au 31 mai 1961, nous avons cherché à les exploiter, car il nous a paru intéressant, disposant de plusieurs années d'observations, de préciser le régime de vidange de la source et de vérifier la validité d'emploi des formules de tarissement habituellement utilisées.

\section{Analyse des courbes annuelles} de tarissement

Afin de pouvoir analyser l'évolution du débit de la Foux-de-la-Vis à l'aide des formules établies pour le régime rigoureusement non influencé, nous avons cherché à déterminer l'influence de certaines pluies parasites de saison sèche sur le comportement de la source. Ainsi nous avons pu constater que les pluies d'étiage, inférieures à $10 \mathrm{~mm}$, et isolées n'influencent pas d'une manière sensible le débit de la Foux. Il suffit cependant que plusieurs pluies voisines de $10 \mathrm{~mm}$ se succèdent pour avoir une action sur le débit. Cette action peut être de deux sortes :

- retardement du tarissement provoquant un décalage dans le temps de la courbe du tarissement, ce qui signifie une recharge sensible de réserves; - action des pluies momentanée, et l'hydrogramme de la nouvelle décrue rattrape la courbe de tarissement au bout d'un temps plus ou moins long (fig. 1).

Dans le cas où l'on n'observe pas une courbe continue de tarissement pour des raisons évoquées 


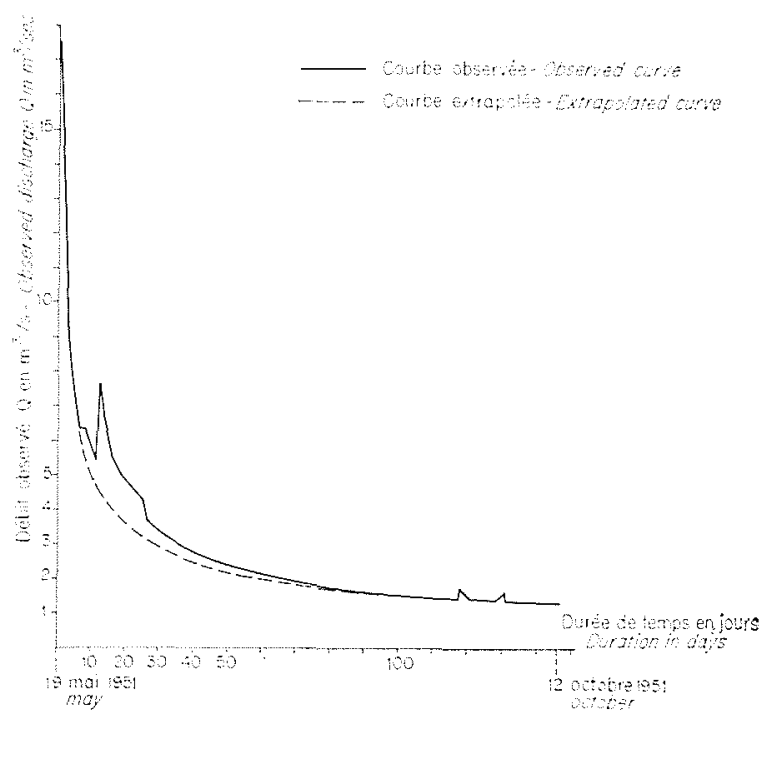

plus haut, on a intérêt à séparer la partie régulière représentant le tarissement pur tel qu'il se serait produit sans l'intervention de la pluie d'étiage, de la partie modifiée sous l'influence de cette pluie.

En superposant les différentes décrues d'une mème année, nous avons obtenu des courbes de tarissement pur que nous appelons « courbes extrapolées de tarissement»; signalons que cette superposition se réalise en général bien au cours d'une mème période de vidange, mais que, par contre il est impossible de trouver une courbe moyenne de tarissement de la Foux-de-la-Vis pour l'ensemble des étiages considérés (1950 à 1961).

La formule de base de courbe de tarissement est celle de Maillet :

$$
\mathrm{Q}_{t}=\mathrm{Q}_{0} e^{-\alpha t}=\mathrm{Q}_{0} e^{-t / \mathrm{T}}
$$

ò̀ : $\mathrm{Q}_{0}$ : débit à l'instant $t=0$;

$\mathrm{Q}_{t}$ : débit à l'instant $t$;

$e: 2,78$ : base de logarithme népérien;

$\alpha$ : coefficient de tarissement de la source égal à $1 / \mathrm{T}$, où : $\mathrm{T}=$ durée de temps nécessaire pour que le débit primitif décroisse dans le rapport de 1 à $1 / e$, soit à 0,368 fois sa valeur initiale.

En coordonnées semi-logarithmiques, cette expression est représentée par une droite dont la pente permet de calculer $\alpha$.

L'intégration de la formule permet d'évaluer le stock d'ear disponible contenu dans les réserves souterraines à un instant $t$, à partir du débit $Q_{t}$ correspondant à cet instant :

Volume du stock:

$$
\int_{t=t_{0}}^{\infty} Q_{t} d t=\int_{t=t_{0}}^{\infty} Q_{0} e^{-\alpha t} d t=\frac{Q_{0}}{\alpha}=\underbrace{Q_{0}}_{\text {nom } t=t_{0}} \mathrm{~T}
$$

\section{Résultats obtenus par application de la formule exponentielle à la Foux-de-la-Vis}

L'application de la formule exponentielle à la Foux-de-la-Vis (fig. 2 et 3 ) montre qu'il n'est pas possible de ramener ici la décroissance du débit en période de vidange à une simple fonction exponentielle. Seules les parties terminales des hydrogrammes obtenus en coordonnées semi-logarithmiques donnent des droites dont les équations montrent une décroissance lente du débit qui correspond bien à la vidange d'un réservoir en régime laminaire. La partie supérieure de l'hydrogramme est une courbe et indique qu'au début de la vidange il y a plusieurs régimes de décroissance des débits qui se superposent au régime laminaire. Théoriquement, il est possible de séparer sur l'hydrogramme en coordonnées semi-logarithmiques les différents composants de l'éconlement. Si on effectue cette décomposition à partir de la partie terminale rectiligne de la courbe de décroissance, on s'aperçoit que la courbe de décroissance considérée correspond assez bien à la superposition de trois droites (fig. 3) dont les coefficients respectifs $\alpha_{1}, \alpha_{2}$ et $\alpha_{3}$ correspondent aux trois différentes sortes d'écoulement et que si l'on veut analyser le tarissement à 
Tableau 1

\begin{tabular}{|c|c|c|c|c|c|c|c|c|c|c|}
\hline Anxpe & 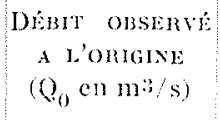 & $\alpha_{1}$ & $V_{0}$ & $a_{2}$ & $\mathrm{v}_{0}{ }^{\prime \prime}$ & $U_{3}$ & $V_{0}^{\prime \prime \prime}$ & $v_{0}$ tot. & $V_{e}$ & $\left(V_{0}-V_{c}\right)$ \\
\hline 1950 & 10,4 & 0,286 & 1,3 & 0.036 & 7,3 & 0.0025 & 52,0 & 60,6 & 31 & 29,6 \\
\hline 1951 & $17, \overline{3}$ & 0,4 & 2,3 & 0,056 & 6,7 & 0,0038 & $54, \overline{5}$ & 63,5 & 31 & 32,5 \\
\hline 1952 & 18,6 & 0,286 & 3,4 & 0,05 & 8,6 & 0,0030 & 65,6 & 77,6 & 39 & 38,6 \\
\hline 1953 & 10,8 & $0, \overline{5}$ & 1,3 & 0,048 & 3,6 & 0,0032 & 37,4 & 42,3 & 21,4 & 20,9 \\
\hline 1954 & 14,4 & $0, \bar{j}$ & 0,9 & 0,063 & 7,6 & 0,0042 & 54,0 & 62,5 & 24,9 & 37,6 \\
\hline 1955 & 24 & 1 & 1,1 & 0,083 & 8,1 & 0,0045 & 61,0 & 70,2 & 20,9 & 49,3 \\
\hline 1956 & 10,3 & 2 & 0,2 & 0,091 & 3,1 & 0,0070 & 43,2 & 46,5 & 16 & 30,5 \\
\hline 1958 & 10 & 1 & 0,3 & 0,091 & 4,7 & 0,0042 & 41,4 & 46,4 & 20,8 & 25,6 \\
\hline 1960 & 22,4 & 2 & 0,4 & 0,143 & $3, \overline{0}$ & 0,0100 & $4 \overline{5}, 8$ & 49,7 & 14,7 & 35,0 \\
\hline
\end{tabular}

partir de la pointe, le débit de la source à l'instant $t$ sera la somme de trois exponentielles :

$$
\mathrm{Q}_{t}=q_{0}{ }^{\prime} e^{-\alpha_{1} t}+q_{0}^{\prime \prime} e^{-\alpha_{2} t}+q_{0}{ }^{\prime \prime \prime} e^{-\alpha_{3} t}
$$

la somme de $\left(q_{0}^{\prime}+q_{0}^{\prime \prime}+q_{0}^{\prime \prime \prime}\right)$ étant égale au débit observé à l'instant $t_{0}$.

Au bout d'un certain temps les premiers termes de l'équation peuvent être négligés (fig. 3). Le volume initial disponible serait done la somme de trois volumes différents :

$$
\begin{aligned}
V_{0}\left(\text { en } \mathrm{m}^{3}\right)=V_{0}^{\prime}+V^{\prime \prime}{ }_{0}+V^{\prime \prime \prime}{ }_{0} & \\
& =\left(\frac{q_{0}{ }^{\prime}}{\alpha_{1}}+\frac{q_{0}{ }^{\prime \prime}}{\alpha_{2}}+\frac{q_{0}{ }^{\prime \prime \prime}}{\alpha_{3}}\right) 86400
\end{aligned}
$$

et le volume en fin de période de vidange serait seulement fonction du débit $q^{\prime \prime \prime}$ et $\alpha_{3}$ et correspondrait à celui de microfissures :

$$
\mathrm{V}_{e}\left(\mathrm{~m}^{3}\right)=\frac{q^{\prime \prime \prime}}{\alpha_{3}} 86400
$$

La différence des deux volumes donnant le volume d'eau écoulé pendant l'étiage :

$$
\text { volume écoulé }=\left(V_{0},-V_{0}\right)
$$

Les tableaux 1 et 2 donnent les coefficients de tarissement des différentes composantes de l'écoulement et les volumes initiaux disponibles $\left(V_{0}\right)$ résiduels en fin d'étiage $\left(V_{c}\right)$ et écoulés pendant l'étiage, pour les amnées étudiées, à l'exception de 1957 et 1959 , les précipitations ayant été trop importantes pendant les étiages de ces deux années pour qu'il y ait en tarissement (le coefficient $\alpha$ ayant l'inverse du temps en jours comme dimension et les volumes étant exprimés en $10^{6} \mathrm{~m}^{3}$ ).

Les pourcentages des différents volumes qui entrent en jeu pour chaque année considérée sont donnés dans le tableau 2.

Ces résultats permettent de préciser le comportement hydraulique de l'aquifere de la Foux-de-laVis :

$1^{\circ}$ ils mettent en relief l'existence de vitesses de décroissance très différentes : à $\alpha_{3}$ correspond une vidange très lente, en régime laminaire, tan-

\begin{tabular}{|c|c|c|c|}
\hline ANNǴE & $\begin{array}{l}v_{0} \\
(\%)\end{array}$ & $\begin{array}{l}V_{0}{ }^{\prime \prime} \\
(\%)\end{array}$ & $\begin{array}{l}v_{0}{ }^{\prime \prime \prime} \\
(\%)\end{array}$ \\
\hline $\begin{array}{l}1950 \\
1951 \\
1952 \\
1953 \\
1954 \\
1955 \\
1956 \\
1958 \\
1960\end{array}$ & $\begin{array}{l}1,5 \\
3,6 \\
4,4 \\
3 \\
1,4 \\
1,6 \\
0,3 \\
0,6 \\
0,8\end{array}$ & $\begin{array}{c}12 \\
10,5 \\
11 \\
8,5 \\
12 \\
11,5 \\
6,8 \\
10,2 \\
7\end{array}$ & $\begin{array}{l}86 \\
36 \\
84,6 \\
88,5 \\
86,5 \\
87 \\
93 \\
89,2 \\
92\end{array}$ \\
\hline
\end{tabular}
dis que les vitesses sont beaucoup plus rapides pour $\alpha_{2}$ (environ 14 fois plus rapides que pour $\alpha_{3}$ ) et pour $\alpha_{1}$ (environ 150 fois plus rapides que
Tableau 2

pour $\alpha_{33}$ ) : nous interprétons ces différences de vitesse comme étant la conséquence de la juxtaposition de plusieurs types de porosité dans le bassin d'alimentation de la Foux;

$2^{\circ}$ les coefficients $\alpha_{1}, \alpha_{2}, \alpha_{3}$, variables d'une année à l'autre, vont grosso modo en augmentant de 1950 à 1960 ; la variabilité de $\alpha$ pouvant être la conséquence d'une hétérogénéité du bassin et d'une alimentation des réserves irrégulière dans le temps el dans l'espace. L'augmentation progressive de ces coefficients pourrait être liée à l'évolution karstique du système, à savoir l'agrandissement et l'augmentation des cheneaux qui joueraient un rôle de plus en plus important dans la circulation;

$3^{\circ}$ en ce qui concerne les volumes, on voit que dans tous les cas e'est le volume $V_{0}{ }^{\prime \prime}$, qui correspond a $\alpha_{n}$, qui est de beaucoup le plus imporlant par rapport à $V_{0}^{\prime \prime \prime}$ et $V_{0}^{\prime}$, constituant de $85 \%(1952)$ à $93 \%$ (1956) du volume total à l'instant initial : nous admettons que ces indications traduisent la prédominance des microfissures dans l'alimentation de la résurgence et nous lions cette porosité à la présence très générale des dolomies du Bathonien (et du Lias) dans le bassin d'alimentation de la Foux-de-laVis;

$4^{\circ}$ ils nous montrent enfin que vu les différentes valeurs des coefficients $\alpha$ observés pour chaque année, il est risqué de vouloir caractériser le régime d'une source par de tels coefficients si l'on ne dispose que d'une année d'observation. 
Aussi, il nous paraît de la plus grande importance, si l'on veut que ces coefficients aient une rigoureuse signification, de les relier de très près au régime pluviométrique d'un bassin, et plus précisément à la répartition dans le temps et dans l'espace des averses sur ce bassin.

Cependant, dans le cas considéré, il n'a pas été possible d'étudier plus en détail les relations pluiesdébits, faute d'une connaissance précise de la pluviométrie réelle du bassin.

\section{Emploi d'une autre formule}

L'application de la formule exponentielle, parce qu'elle nécessite la décomposition de l'hydrogramme observé, est dans le cas de la Foux-de-la-Vis d'un usage peu commode : en efít, cette décomposition ne peut être faite que lorsque l'on connait la partie terminale de l'hydrogramme. Aussi avons-nous procédé à la recherche d'une autre formule (Cf. H. Schoeller, 1948). En étudiant la relation qui existe entre $d q / d t$ et $q$ qui ne nous a pas donné de résultat, et ensuite entre $\log (d q / d t)$ et $\log q$, nous arrivons à une formule du type :

$$
\frac{1}{\mathrm{Q}^{1 /(n+1)}}=\frac{1}{\mathrm{Q}^{1 /(n+1)}}+\beta t
$$

elle correspond à une nappe libre en régime mixte ou turbulent. Dans notre cas, $n$ serait égal environ $\dot{a}-0,5$ ce qui domerait :

$$
\begin{aligned}
\frac{1}{Q_{i}{ }^{2}}=\frac{1}{Q_{0}{ }^{2}}+\beta t \quad \text { ou } \quad Q_{i}= & \frac{1}{\left[\left(1 / Q_{0}{ }^{2}\right)+\beta t\right]^{1 / 2}} \\
& =\frac{Q_{0}}{\sqrt{1+Q_{0}{ }^{2} \beta t}}
\end{aligned}
$$

$Q_{0}$ et $Q_{t}$ étant les débits observés aux instants $t_{0}$ et $t$, et $\beta$ un coefficient de décroissance du débit.

Cette équation donne une droite en diagramme arithmétique si l'on porte :

- en ordonnées, l'inverse du débit observé au carré;

- en abscisses, le temps correspondant.

La pente de la droite permet de calculer $\beta$ (fig. 4).

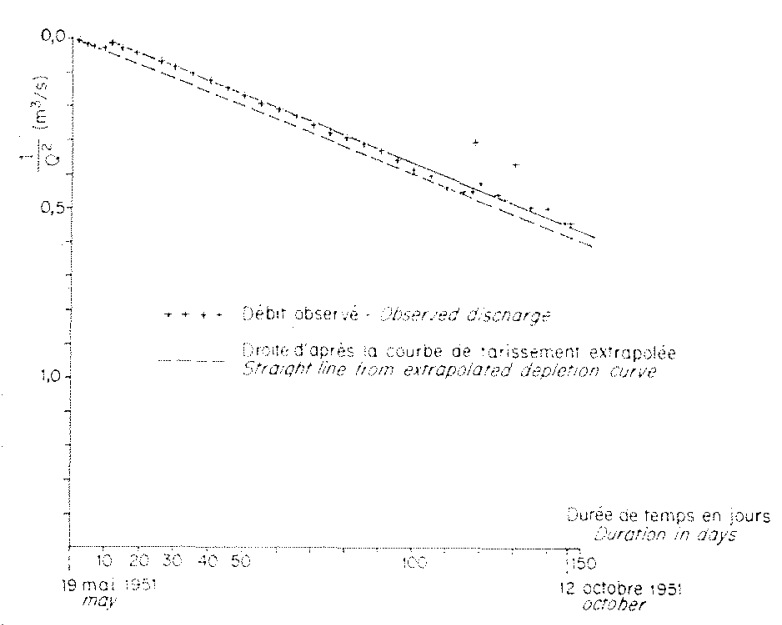

4/
L'intégration de la formule entre deux instants donnés donne le volume d'eau écoulé pendant cet intervalle :

$$
\int_{t=t_{0}}^{t} Q_{t} d t=\frac{2}{\beta}\left(\begin{array}{cc}
1 & 1 \\
Q_{t} & Q_{0}
\end{array}\right)
$$

done :

\begin{tabular}{|c|c|c|c|}
\hline AnNí: & $Q_{0}$ & $\beta$ & $\begin{array}{l}\left(V_{0}-V_{e}\right) \\
\left(e n 10^{\prime \prime} \mathrm{m}^{3}\right)\end{array}$ \\
\hline $\begin{array}{l}1950 \\
1951 \\
1952 \\
1953 \\
1954 \\
1955 \\
1956 \\
1958 \\
1960\end{array}$ & $\begin{array}{l}10,4 \\
17,5 \\
18,6 \\
10,8 \\
14,4 \\
24,0 \\
10,3 \\
10,0 \\
16,7\end{array}$ & $\begin{array}{l}0,0059 \\
0,0040 \\
0,0034 \\
0,0093 \\
0,0037 \\
0,0028 \\
0,0037 \\
0,006 \\
0,0041\end{array}$ & $\begin{array}{l}30 \\
32 \\
36 \\
21 \\
36 \\
46 \\
31 \\
26 \\
15\end{array}$ \\
\hline
\end{tabular}

$$
\left(V_{0}-V_{c}\right) \text { en } 11^{3}=\frac{2}{\beta}\left(\frac{1}{Q}-\frac{1}{Q_{0}}\right) 86400
$$

Le tableau résume les résultats obtenus:

\section{Tableau 3}

En ce qui concerne $\beta$, les valeurs sont variables aussi d'une année à l'autre, mais cette variabilité semble être irrégulière. Les raleurs des volumes d'eau écoulés sont comparables à celles obtenues par la formule exponentielle. Ainsi, cette formule, beaucoup plus simple, done d'usage plus facile, que la formule exponentielle, a l'avantage d'être la même pour toute la période de vidange. Elle permet de prévoir le débit de la source en fonction du aćbit $Q_{0}$ observé à l'instant $t_{0}$, en ne connaissant que le début de la courbe de vidange, ceci bien sûr en régime non influencé.

En ce qui concerne l'appréciation des volumes, l'intégration de la formule permet de calculer le volume d'eau écoulé entre deux instants, et seulement lorsque la durée entre ces deux instants est assez longue. Par contre, elle ne permet pas de calculer le volume emmagasiné à un instant donné, l'utilisation de la formule antre un temps donné et l'infini donnant toujours un rolume infini et c'est là son principal inconvénient.

Bibliographie

C. Drogue (1964) : Etude géologique et hydrométrique des principales résurgences de la région nord-montpellieraine : sources du Lez, du Lirou et de Sauve. Thèse $3^{3}$ cycle, C.E.R.H. Montpellier (avril 1964).

H. PAloc (1962) : Contribution à la connaissance des circulations karstiques dans une région type du midi méditerraneen. Observations sur le comportement aquifère des dolomies, mémoires de I'A.I.H. tome $V$, réunion d'Athenes, pp. 243-248.

(1964) : Carte hydrogéologique de la région karstique nordmontpelliéraine a l'échelle du $1 / 80000$. Foux-de-la-Vis : numéro 211.

H. Schonller (1948) : Le régime hydrogéologique des calcaires éocènes du synclinal du Dyr-El-Kef (Tunisie). Bull. Soc. Géol. Fr., 5e série, t. 18 , p. 167 à 180 .

(1962) : Les eaux souterraines. Edition Masson \& Cie, Paris, एр. 205 à 214 . 


\section{Discussion}

Président : M. Nicoras

M. le Président remercie M. Paloc d'avoir présenté cette communication et remarque que, d'après je tableau inclus dans le rapport, les coefficients $\alpha_{1}, \alpha_{2}, \alpha_{3}$, variables d'une année it l'autre, vont très grosso modo en augmentant de 1950 a 1960. Xl. Paloc peut-il donner une explication de ce phénomène, bien qu'il s'agisse en fait d'un très petit nombre de cas?

i. Paloc dit que, malgré leur faible nombre, ees chiffres traduisent une évolution peut-être plus large a l'échelle de la décade, mais posent bien le problème du tarissement progressif des sources karstiques.

C'est ainsi que, notamment pour la fontaine de Vaucluse, on a constaté qu'il y a près d'un siècle, le déversement s'effectuait par la vasque supérieure pour une valeur de débit qui était de l'ordre de $21 \mathrm{~m} 3 / \mathrm{s}$, Aetuellement ce déversement semble n'avoir lieu que pour une valeur bien supirieure du débit, voisine de $30 \mathrm{~m}^{3} / \mathrm{s}$. Dans l'intervalle, il semble que se soit produite non pas tellement ine diminution du régime pluviométrique ayant engendré une baisse généralisée des plans d'eau, mais plutot une évolution des caractéristiques des exutoires liée a l'éroluition karstique, à savoir que les eaux souterraines ont de plus en plus tendance à agrandir les canaux les plus inférieurs du système.

En re qui conceme la Foux-de-la-Vis, le B.R.G.M. ne possede que dix ans d'observations, done pas asse\% de recul et, surtout pas un nombre suffisant de points de comparaison avec d'autres systèmes en France pour pouvoir donner des conclusions précises quant a cette évolution :

Le B.R.G.M. connait assez mal quelle a été la répartition de la pluie sur le bassin versant, laquelle est caprieicuse dans celte région climatique a influence méditerranéenne. Il n'a pas été possible de ramener à une valeur homogène pur l'ensemble du bassin les pluies observies pour une même période d'observation. Les valeurs différentes de peuvent provenir du fait que, selon les amees, des régons du bassin de porosités difféentes recoivent plus de pluie et contribuent d'une manière prépondérante a l'alimentation de la source.

M. Je Président pense que le coefficient a donne une idée de la nature des fissures et est lié par consérquent à leur développement.

M. Thmmot remarque que, puisque le phénomène est régulier, il faudrait supposer que la répartition de la pluviometrie se fait de façon uniforme d'un enchroit à l'autre et que ce coefficient a indiquerait peut-ître une modification du régime. Mais ce n'est pas d'une si courte période d'observation qu'on peut tirer une conclusion valable.

M. Thinriot se demande, d'autre part, si on ne peut pas penser à une corrélation entre les valeurs du débit et les coefficients $a$. Les régions à porosite variable dépendent, peut-être, aussi de la valeur du débit et on pourrait vérifier ceci en comparant les pentes de denx courbes de tarissement au droit d'une ordonnée correspondant à un même débit. Est-ce que le résultat irait dans le sens d'une relation déterministe entre le débit et le coefficient $\alpha$, plutôt que dans le sens d'une relation probabiliste qui serait due aux variations des précipitations?

M. Paloc précise que les travaux, objet de la communication présentée, consistaient à préciser par un argument supplémentaire les propriétés aquifères de la dolomic, à savoir la prédominance des microfissures, dans l'alimentation de la source. Il reste à comparer les courbes de tarissement non plus effectivement d'après l'origine des temps, mais d'après une même valeur du débit à différentes epoques, comme le suggère M. Thinniot.

M. Guillot remarque, sur la forme, que le remplacement du coefficient $\alpha$ par $1 / 0$ permet de comparer plus commodiment les vitesses de décrue. En effet, - $\theta$ est l'inverse de la dérivée logarithmique du débit par rapport au temps et $1 / \theta$ donne une idée de la déerue journalière du débit en valeur relative. $\theta$ s'exprime en jours : pour $\theta=20$ jours, le débit décroît de $5 \%$ d'un jour à l'autre; pour $0 / 40$ jours, le débit décroit journellement de $2,5 \%$.
Sur le fond, M. Gullot souligne que $\theta$ croît avec le temps, en règle très genérale pour toutes ces rivières et pas senlement pour les sources karstiques : une courbe de tarissement peut ètre representée par plusieurs troncons qui correspondent à des valeurs de $\theta$ croissantes au fur et a mesure que le debit diminue.

M. Gullor estime, enfin, que les débits des rivières du Jura et des Préalpes (Doubs, Ain, Yome), qui sont mesurés correctement depuis des dizaines d'annees grâce à l'industrie hydroćlectrique, correspondent á la somme du débit de quelques sources karstiques, à moins que l'on $y$ observe encore un écoulement superficiel deux jours après la fin de la pluie, ce qui apparemment n'est pas le cas. Les constantes de temps de ces rivières peuvent donc ètre mises en comparaison avec celles que Mme Forkasiewicz et M. Paloc obtiennent en observant les débits de la Foux-dela-Vis.

M. Paloc indique qu'il a exprimé la formule sous dit forme Q eat mais qu'en réalité Mme Fonkasiewicz et Jui ont toujours remplace $\alpha$ par $1 / \theta$ et indiqué les durées en jours dans leurs calculs.

Mme Fonsasmwicz et M. Paloc sont, d'autre part, bien d'accord sur la validité de la loi du tarissement pour tous les réservoirs, qu'ils soient ou non karstiques. Mais, en tant que naturalistes, ils cherchent à illustrer par une formule commode ce que révèlent les arguments de faciès, de géchimie, de pouvoir d'attaque, de transport et même de variations de temperature, quant à l'intérêt d'une telle source karstique du point de vue des réserves. Pour la Fouxde-la-Vis, les observations de Mme Fonkasiewicz et de M. Paloc ains1 que l'application de la formuje mentionnéc dans leur memoire ont permis de rendre compte de façon satisfaisante du comportement de l'aquifère.

Toutefois, M. Paloc ne croit pas que le débit d'une rivière comme l'Ain, puisse traduire les caractéristiques de chacun des exutoires des divers faciès ealcaires drainés par cette rivière, En effet les coefficients de decrue observés montrent des différences importantes d'une source à l'autre: $10^{-6} \mathrm{I} / \mathrm{s}$ à la source de Sauve (Gard) et $10^{-8} \mathrm{l} / \mathrm{s}$ à la Fonxde-lat-Vis. Si l'on jauge l'écoulement total a l'aval de telles résurgences, on n'aura qu'une indication sur le comporte. ment global du milieu karstique. Or, ce que l'on recherche à ménager, ee n'est pas d'ensemble mais l'une ou l'autre de ces résurgences.

En tout cas, dans le fura comme ailleurs, il y a plusieurs faciès qui participent à l'écoulement total el il est bien certain qu'il est préférable de procéder ponetuellement que de se fier a des jaugeages globaux. Pour atteindre ce but il faut multiplier les equipements sur les sources.

M. BANAL s'associerait volontiers an sounait d'accrôtre les points d'observation des sources, mais signale que souvent les observations s'accumulent sans ètre exploitées autant qu'elles le pourraient. Dans le cas particulier, M. Banal se demande si on ne pourrait pas aller plus loin dans l'interprétation des constatations faites sur les courbes de tarissement, et demande à M. Gurrot comment il aborderait le problème.

M. Guncot explinue que le tarissement doit être étudié sur des irtervalles de temps non influencés, c'est-à-dire se situant entre deux periodes pluvieuses. On trace ainsi sur un graphique les tronçons de droites représentant le logarithme du débit en fonction du temps (fig. 1). L'ensemble de ces tronçons correspond a une masse de points $(q, \theta)$ assez dispersés (fig. 2), et l'on constate que les valeurs fortes de $\theta$ correspondent à des débits faibles, et inversement.

D'autre part, il est manifeste que $\theta$ ne dépend pas seulement du débit, mais aussi du delai depuis la demiere pluie, car, deux ou trois jours après la fin de l'averse, il n'y a plus de ruissellement, et malgré cela on a une déerue rapide qui semble rejoindre le niveau de la déerue antérieure (M. Paloc le retrouve d'ailleurs). Ce niveau ne s'est beaucoup èlevé que si la pluie a été suffisamment longue et importante (fig. 3 ). 

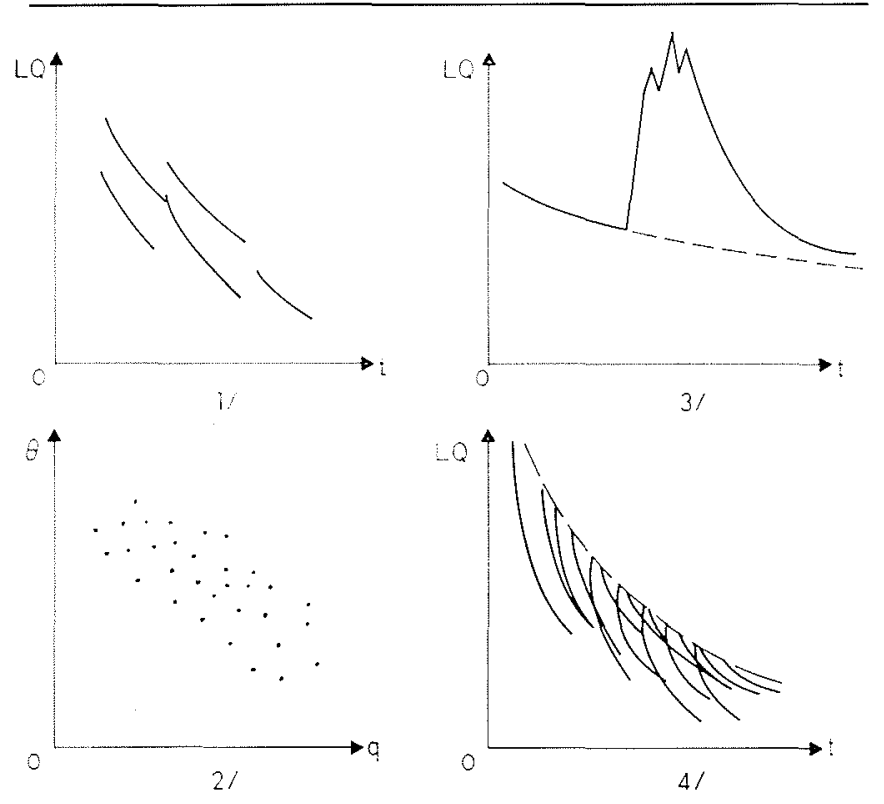

Finalement, en collectionnant les valeurs de $\theta$, on arrive à l'idée qu'il y a non pas une courbe de décrue, mais une population de courbes de décrue, et qu'elles sont toutes en dessous d'une décrue qui correspondrait a un départ avec terrain totalement saturé et en restant a chaque fois en rẻgime «éguilibré », c'est-á-dire avec les couches supérieures à vidange rapide se vidant les premières et les couches inférieures se vidant ensuite. Cette décrue constitue en somme la «décrue enveloppe» (fig. 4), la décrue la plus lente possible.

Quand on part d'un débit donné, on n'est généralement pas en régime «équilibré ». La dernière pluie a saturé les couches superficielles, mais si l'épisode n'a pas été très long elle n’a pas saturé les couches inférieures, aussi la courbe descend plus vite que la courbe-enveloppe, et on n'obtient que plus tard le régime équilibré.

En collectionnant les courbes de decrue, on arrive a tracer lat conrbe enveloppe. C'est une autre manière de la tracer.

Pour répondre à une question posée tout à liheure, M. Gulloot ne pense pas qu'il y ait me liaison entre le débit et le $\theta$; simplement, pour un débit donné, il y a un $\theta$ maximal possible.

Le débit n'est une image des réserves souterraines que si lon se place suffisamment longtemps avant la pluie. On la constate d'ailleurs dans le rapport de M. Paloc.

Puisqu'il est évident que le $\theta$ augmente avec le debit, il est compréhensible qu'on soit tenté d'ajuster à un schéma conme celui que M. Paloo propose et dans lequel $1 / q^{2}$ est une fonction lincaire du temps (parce que cela donne un 0 qui augmente avec $t$ ). Dans ce cas on a $\theta=2 t$ (l'inverse de Ia dérivée logarithmique du débit par rapport au temps est égal à $2 \%$. On a une constante qui augmente avec la durée de la décrue.

M. Gumlon se demande si le fait que M. Paloc arrive a décrire les décrues d'un seul été par une seule courbe en $q^{-2}+\beta t$ correspond, non à un phénomène physique, mais plutôt à la description statistique du phénomène suivant; au cours d'un seul été, toutes les décrues sont de plus en plus lentes et de plus en plus basses et, en les mettant bout a bout, on arrive à former une courbe où les $\theta$ augmentent. Le fait que la constante de temps varie d'un été à l'autre pent dépendre, soit du niveau général d'humidité initiale, soit des caractéristiques aléatoires de l'été considéré.

M. Gurbot suggère, en conclusion, de tenter d'établir, pour la Foux-de-la-Vis, une corrélation entre le niveau des étiages aux mois d'août et de septembre et de la pluviosité de l'hiver piécédent. Cette corrélation apparait implicitement dans le rapport de M. PaLoc puisqu'il $y$ est indiqué que $\theta$ est déterminé dès le début de la décrue; donc le niveau des etiages est, au bout d'un temps un peu long, tel que :

$$
q=\beta / \sqrt{ }^{T}
$$

Le dẻbit d'étiage étant ainsi déterminé dès le début de la décrue, il doit exister une liaison entre les débits d'étiage el la pluviosité de l'hiver précident.

M. le professeur Avis pense que la resherche de la corrélation suggére par M. Guruor serait certes tres utile mais ne suffirait pas. La localisation des précipitations (averses), surtout a l'échelle d'une année, jone en effet un grand rôle dans la région considérée vu l'hétérogénéité géologique. Il ne faut, en effet, pas oublier que nous sommes en région de climat méditeranéen on marriméditerranéen, donc à précipitations très localisées et condensées dans lo temps; par ailleurs, que le bassin d'alimentation de la Fouxde-la-Vis est de superficie réduite, ce qui réduit la régularisation statistique. On peut assister, en Languedoc, a des précipitations exceptionnelles dans certains bassins alors que dans des bassins adjacents on aura des conditions exceptionnellement sèches pendant la même période. Le cas s'est par exemple présenté en 1965 pour le bassin de Ja source du Lez (qui alimente Montpelliel en cau potable) Cette dernière source a présenté un étiage exceptionne (moins de $450 \mathrm{l} / \mathrm{s}$ alors que dans le Languedoc en général on a cu une année exceptionnellement humide. En conclusion, l'irrégularité des courbes de tarissement de la Foux-dela-Vis semble, du point de vue naturaliste, tout a fait normale.

M. Gurmór veut bien qu'il y ait hétérogénéité des averses quotidiennes on mème hebdomadaires, mais on ne croit pas qu'il y ait, sur la précipitation globale du mois de novembre atu mois de mars, une dispersion telle qu'il serait vain, parce que certains points sont spécialement arrosés d'une annéc et d'autres pas, et inversement pour d'autres années, de chercher une liaison entre la pluviosité de l'hiver et l'étiage l'été. Il y a un moven de lever le doute : c'est d'examiner le rapport entre les precipitations mesurées en divers pluviometres du bassin.

M. Thumbor remarque que, de toute façon, si l'on ne considere que de petits tronçons de courbe de tarissement, on suit des périodes courtes de pluviosité, ce qui peut ramener a une hétérogénéité a une échelle de temps plus courte que les quatre mois considérés par M. Gunxor.

M. Gullot pense que, si la réserve soutermine en fir d'hiver détemine en pratique le plancher des étiages d'été, il doit y avoir une liaison entre la pluie d'hiver et l'étiage d'été.

M. le Président conclut qu'il faut faire la comparaison pluviométrique suggerée par M. Gullot, Jaquelle n'est pas très difficile.

II. Guros fait une remarque concernant la loi de tarissement d'une nappe, dans le cas général. La théorie et des expériences faites sur des réseaux d'assainissement montrent que, pour des nappes situées dans des terrains «perméables en petit $»$, la loi de tarissement dépend de la morphologie de la nappe et en particulier de la situation du fond imperméable par rapport an niveau des exutoires. Analyliquement, la loi exponentielle est un cas limite, asymptotique (Boussinesq 1904; études du C.R.E.C.G.R., 1959-1966).

M. Paloc rappelle que M. Schoellen, en comparant tous les types d'aquiferes possibles, est arrivé à la conclusion que la formule exponentielle etait valable dans la mesure ou l'exutoire ne présentat pas de variations de niveau importantes.

Mme Forisievic ajoute que la formule exponentielle convient pour la partie terminale, mais non pour le début du tarissement. Mme Forkasiewicz et M. PaLOC ont done cherché, pour la première partie, une autre formule en appliquant la variation de la pente en fonction du débit.

M. le Président croit que la remarque de M. Guyon est tres pertinente : une formule exponentielle est certainement meilleure lorsqu'on est sous une forme adiabatique; mais dans la mesure ò survient un faux équilibre en cours d'écoulement, elle ne doit certainement plus correspondre à la réalité. Physiquement parlant, c'est un phénomène guénéral.

Il est certain que $\theta$ ne peut dépendre que du débit; il y a d'autres phénomènes qui interviennent suivant qu'on est plus ou moins proche de l'équilibre ou qu'on a une masse d'eau plus ou moins importante à écouler.

M. RÉminfras rejoint les conclusions de M. Gurlor, mais peut-être par une voie différente:

« La formule de Maillet n'est valable qu'en régime noninfluence. Or, au cours des essais effectués dans la nature, on ne sait pas très bien si l'on est en régime influencé ou non-influencé, Dans ces conditions, la décomposition en trois droites de la courbe de tarissement sul le diagramme 
semi-logarithmique de M. PALoc pourrait être analogue à la séparation, dans un hydrogramme de surface, des composantes relatives au ruissellement, à l'écoulement hypodermique et à l'écoulement souterrain. Il n'est pas exclu, en effet, qu'il y ait une part d'écoulement hypodermique (voire de surface) dans les courbes interprétées par M. Paloc.

« D'autre part, le procédé utilisé par les auteurs pour tracer, à partir des relevés expérimentaux, la courbe de tarisscment moyenne parait à $M$. RÉmb́ní́ras assez discutable. Comme le remarque $\mathrm{H}$. Guillor, on ne peut obtenir, sur le terrain, que des tronçons de la courbe ci-dessus et toujours avec une dispersion assez grande; nous avons tenté de réduire celle-ci en traçant deux courbes de tarissement; l'une pour la saison chaude et l'autre pour la saison froide. Daus certains cas on peut penser, en effet, qu'au cours de la période de tarissement, la réserve souterraine alimente, non seulement le débit mesuré dans le cours d'eau, mais également ume partie de l'évaporation du sol et des plantes, dans tout ou partie de son bassin versant. 》

M. Baral constate que la discussion met bien en évidence les points de vue apparemment contradictoires de ceux qui jugent les phénomenes trop compliqués pour que leur représentation par des formules puisse même être envisagée, et ceux qui recherchent des ajustements de plus en plus précis.

Il ne faut pas attacher, a priori, une valeux physique à l'utilisation de telle ou telle fonction et la fonction exponentielle a peut-être sa simplicité pour seule vertu; mais la représentation d'une suite de mesures par la valeur d'un coefficient est toujours un moyen d'étude très puissant.
La confrontation a laquelle nous assistons est certainement très utile.

M. Castany rappelle encore un point à considérer: : c'est qu'on est en terrain calcaire, et qu'il $y$ a done deux facteurs qui jouent dans le comportement du réservoir situé dans ce terrain:

- l'altitude du plan d'eau, qui dépend de la pluviométrie de l'année précédente et réagit sur la hauteur de charge a l'exutoire;

- la quantité d'eau stockée par tranche de calcaire, qui diffère selon l'hétérogénéité du terrain : dans le massif de Ben-Saidane, or a constaté que le premier mètre de roche réservoir contenait $750000 \mathrm{~m}^{3}$ et le second $500000 \mathrm{~m}^{3}$. Au Chennata, le volume d'cau emmagasine décroît en profondeur de 27000 à $13000 \mathrm{~m}^{3}$ par mètre d'abaissement.

Pour faire des prévisions en Tunisie entre la pluviométrie d'une année et le débit possible pour l'année suivante, il a fallu établir une corrélation en tenant compte de l'altitude du plan d'eau au début de la saison de recharge, qui dépend de la pluviométrie de l'année précédente, et de l'altitude du plan d'eau à la fin de cette même saison.

M. le Président conclut que, d'une manière générale, en tentant de mettre en équation tous les phénomènes observés, on pose plus de problèmes qu'on n'en résout. C'est sans doute une bonne chose, car c'est ainsi que l'on parviendra peut-être à mieux comprendre les faits observés. Pour l'instant, nous sommes au début des interplétations, les théories devront être soumises à l'épreuve de l'expérience. 


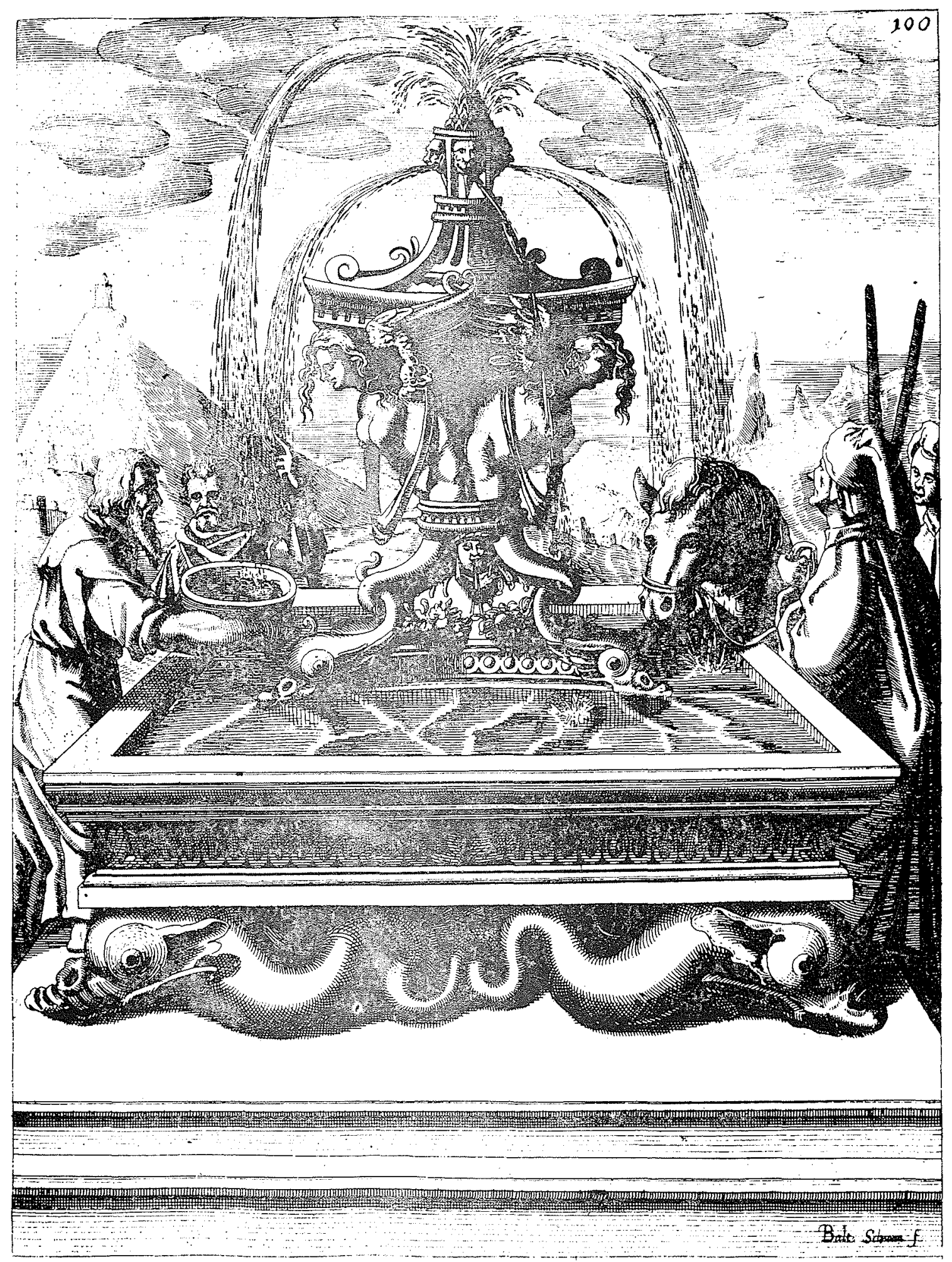

Gravure extraite de Architectura curiosa nova par G. A. BÖCKLERN Nuremberg (1664) 